\title{
Urinuntersuchung beim Kleinsäuger - so einfach und doch so aussagekräftig
}

Jutta Hein

Obwohl die Urinuntersuchung beim Kleinsäuger einfach und kostengünstig durchzuführen ist, wird sie doch viel zu selten in der Praxis genutzt. Dabei liefert sie schnell Antworten sowohl auf typische Fragen (z. B. Blut im Urin oder Kalziumstatus) als auch auf überlebenswichtige prognostische Fragen (wie Nierenfunktion und Säure-Basen-Haushalt) und kann Hinweise auf systemische Krankheiten geben (z. B. Diabetes mellitus und Ketoazidose).

\section{Uringewinnung}

Auch bei Kleinsäugern kann Urin durch verschiedene Methoden gewonnen werden:

- Auffangen freiwillig abgesetzten Urins

- Auffangen nach manuellem Ausdrücken der Blase

- Katheterisierung

- Zystozentese

Welche Methode wann gewählt wird ist von der Fragestellung abhängig ( Tab.1), das benötigte Material zur Urinentnah- me und -untersuchung ist in $\mathbf{A b b} \mathbf{1}$ dargestellt.

\section{Auffangen ohne Manipulation}

Das Tier wird in eine Box mit nicht saugfähiger Perlstreu oder alternativ in eine Box mit Gitterboden über einer sauberen Schale gesetzt. Der Gitterboden verhindert eine Kontamination des Urins mit Kot oder durch Durchlaufen.

\section{Auffangen nach Ausdrücken der Blase} Der Oberkörper des Tieres wird angehoben und der Blaseninhalt aufgeschüttelt und sanft ausmassiert (Vorsicht bei übervoller Blase und/oder Verdacht auf Verschluss), Abb.2. Die Mittelportion ist am repräsentativsten für den Gesamturin, die Anfangsportion eher für die ableitenden Wege.

\section{Katheterisierung}

Die Katheterisierung erfolgt möglichst steril mittels eines Katerkatheters oder einer schmalen Ernährungssonde. Das Tier befindet sich in Rückenlage, ggf. ist eine Sedation notwendig.

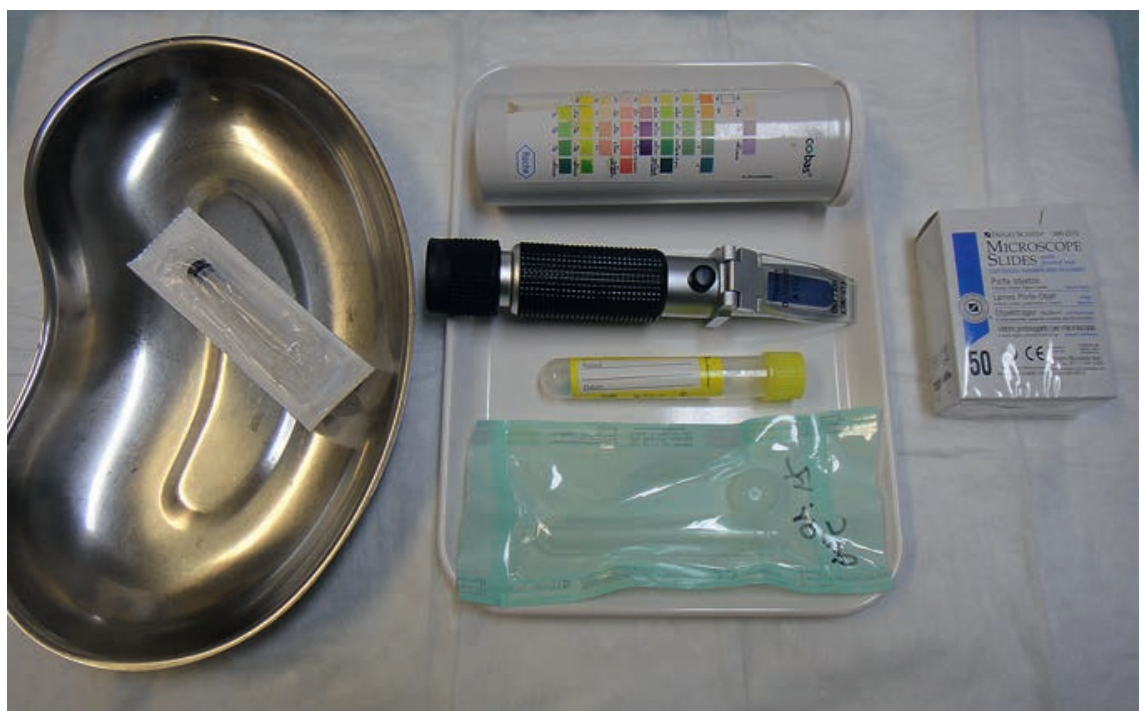

Abb. 1 Material zur Urinentnahme (Auffangschale, sterile Spritze mit Kanüle [22G], sterile Röhrchen mit und ohne Stabilisator), Urinteststreifen, Refraktometer, Objektträger für die Sedimentuntersuchung.

\section{Zystozentese}

Das Tier wird in Rückenlage fixiert, die Punktion erfolgt im Winkel von $30^{\circ}-45^{\circ}$ mit einer kleinen Kanüle (22 G). Die Zystozentese kann blind durch Fixation der Blase zwischen den Fingern ( $\triangleright$ Abb.3) oder unter Ultraschallkontrolle durchgeführt werden.

\section{Vorbereitung der Urinprobe}

Die Urinproben werden in sterilen Röhrchen aufgefangen und möglichst rasch untersucht. Da es besonders bei höheren Temperaturen schnell zur Dissoziation von Zylindern, osmotischer Zellschädigung, Präzipitation von Kristallen und pH-Änderung kommt, sollte Urin nicht länger als $30 \mathrm{Min}$. bei Zimmertemperatur und maximal 48 Stunden im Kühlschrank $\left(+4{ }^{\circ} \mathrm{C}\right)$ gelagert werden. Vor der Untersuchung wird der Urin wieder auf Raumtemperatur gebracht, da manche Tests - z.B. Teststreifen und urinspezifisches Gewicht (USG) - temperaturabhängig sind und Kältepräzipitate sich erst wieder auflösen müssen [10].

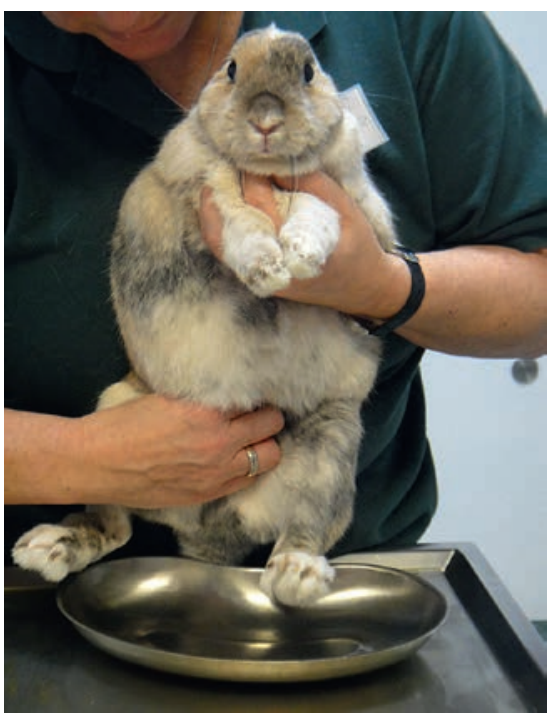

Abb. 2 Manuelle Blasenentleerung beim Kaninchen. 
Tab. 1 Uringewinnung.

\begin{tabular}{|c|c|c|c|c|}
\hline Methode & Vorteile & Nachteile & Geeignet & Nicht geeignet \\
\hline $\begin{array}{l}\text { Auffangen } \\
\text { ohne } \\
\text { Manipulation }\end{array}$ & $\begin{array}{l}\text { keine Manipulation } \\
\text { notwendig }\end{array}$ & $\begin{array}{l}\text { - ggf. zeitaufwendig } \\
\text { Kontamination durch harnablei- } \\
\text { tende Wege und Umgebung } \\
\text { unvollständige Blasenentlee- } \\
\text { rung (ggf. Unterschätzung der } \\
\text { Kristallurie) }\end{array}$ & $\begin{array}{l}\text { makroskopische } \\
\text { Untersuchung } \\
\text { (Blut, Bei- } \\
\text { mengungen, Ca) } \\
\text { - USG } \\
\text { - Urinteststreifen }\end{array}$ & $\begin{array}{l}\text { spezielle } \\
\text { Nieren- und } \\
\text { Blasen- } \\
\text { beurteilung } \\
\text { - Sediment } \\
\text { BU }\end{array}$ \\
\hline $\begin{array}{l}\text { Auffangen } \\
\text { nach } \\
\text { Ausdrücken } \\
\text { der Blase }\end{array}$ & $\begin{array}{l}\text { - schnell und einfach } \\
\text { bei Kleinsäugern geringe } \\
\text { Gefahr von Ruptur und } \\
\text { Rückstau in das Nieren- } \\
\text { becken } \\
\text { - gute Durchmischung und } \\
\text { Entleerung der Blase } \\
\text { - Beurteilung von Nieren- } \\
\text { funktion und Stoffwechsel }\end{array}$ & $\begin{array}{l}\text { Kontamination durch } \\
\text { harnableitende Wege und } \\
\text { Umgebung } \\
\text { - Blutbeimengungen durch } \\
\text { Läsionen v.a. bei Kristallurie }\end{array}$ & $\begin{array}{l}\text { makroskopische } \\
\text { Untersuchung } \\
\text { (Beimengungen, } \\
\text { Ca, nicht für } \\
\text { Blut!) } \\
\text { - USG } \\
\text { Urinteststreifen }\end{array}$ & $\begin{array}{l}\text { spezielle } \\
\text { Nieren- und } \\
\text { Blasen- } \\
\text { beurteilung } \\
\text { - Sediment } \\
\text { BU }\end{array}$ \\
\hline Katheterisierung & $\begin{array}{l}\text { - Überprüfung der Harn- } \\
\text { röhrendurchgängigkeit } \\
\text { Blasenspülung und retro- } \\
\text { grade Eingabe (Kontrast- } \\
\text { mittel, Medikamente) }\end{array}$ & $\begin{array}{l}\text { schwieriger bei weiblichen Kanin- } \\
\text { chen (gemeinsamer Urethra- und } \\
\text { Vaginalausgang) und männlichen } \\
\text { Meerschweinchen (große Samen- } \\
\text { blasendrüsen) } \\
\text { Gefahr der Einschleppung von } \\
\text { Keimen in die Blase und der } \\
\text { Schleimhauttraumatisierung }\end{array}$ & $\begin{array}{l}\text { makroskopische } \\
\text { Untersuchung } \\
\text { (Beimengungen, } \\
\text { Ca, nicht für } \\
\text { Blut!) } \\
\text { - USG } \\
\text { - Urinteststreifen }\end{array}$ & $\begin{array}{l}\text { reine Nieren- } \\
\text { und Blasen- } \\
\text { beurteilung } \\
\text { - Sediment } \\
\text { BU }\end{array}$ \\
\hline Zystozentese & $\begin{array}{l}\text { - schonende Gewinnung } \\
\text { sterilen Blasenurins für BU } \\
\text { - Entleerung der Blase bei } \\
\text { Harnröhrenverschluss }\end{array}$ & $\begin{array}{l}\text { Ruptur bei übervoller Blase } \\
\text { möglich } \\
\text { geringe Blut- und somit auch } \\
\text { Proteinbeimengungen durch die } \\
\text { Punktion }\end{array}$ & $\begin{array}{l}\text { alle Untersuchun- } \\
\text { gen }\end{array}$ & $\begin{array}{l}\text { Nachweis von } \\
\text { Blutspuren }\end{array}$ \\
\hline
\end{tabular}

Für die Untersuchung von Urinstatus und Sedimentes ist frischer, nativer Urin (unbehandeltes, steriles Röhrchen) am besten geeignet. Urinproben für die mikrobiologische Untersuchung sollten in Stabilisatorröhrchen (Röhrchen mit Borsäurepulver) verschickt werden.

\section{konkret}

Urin sollte nicht länger als $30 \mathrm{~min}$ bei Zimmertemperatur und max. $48 \mathrm{~h}$ im Kühlschrank $\left(+4^{\circ} \mathrm{C}\right)$ gelagert werden.

Für die Urinuntersuchung stehen folgende Methoden zur Verfügung:

- Urinstatus (makroskopische Untersuchung, USG, Urinteststreifen)

- Sedimentuntersuchung

- bakteriologische Untersuchung (BU)

- Untersuchung auf andere Infektionserreger (z.B. E. cuniculi-Sporen, Leptospiren)

- Bestimmung von bestimmten klinisch-chemischen Parametern
(z.B. Urin-Kortisol/Kreatinin-Quotient [UCC], Urin-Protein/Kreatinin-Quotient [UPC]).

\section{Urinstatus}

\section{Makroskopische Urinuntersuchung}

Die makroskopische Urinuntersuchung gibt bereits erste wesentliche Hinweise auf Nierenfunktion und Hydratationsstatus (Konzentration und Farbe), auf Stoffwechselfunktion und Säure-BasenHaushalt (Geruch) und auf fütterungsbedingte und/oder krankhafte Beimengungen (Blut, Kalzium, Eiter etc.).

\section{Farbe}

Bei gesundem Urogenitaltrakt sollte ein gut konzentrierter (kräftig gelb gefärbter) Urin abgesetzt werden ( $\triangleright$ Abb.4). Schwach gefärbter Urin spricht für Polyurie (Verifizierung durch Bestimmung des USG). Bestimmte Medikamente, Futterfarbstoffe und andere Pigmente aus dem endogenen Abbau (Gallepigmente, Porphyrine, Melanin etc.) können die
Harnfarbe erheblich beeinflussen (Nachdunkeln, Fehlinterpretation als Blut, - Abb. 5).

\section{Geruch}

Physiologischer Urin sollte aromatisch riechen. Bestimmte Medikamente, Futterfarbstoffe und andere Pigmente aus dem endogenen Abbau können den Geruch beeinflussen (z.B. „Spargelgeruch“). Ein stechender Geruch deutet auf eine Infektion hin, ein fruchtiger Geruch ggf. auf eine Ketose.

\section{Trübung}

Eine Trübung entsteht durch Beimengungen (Kristalle, Schleim, Eiter, Blut, Epithelien, Bakterien). Eine geringgradige Beimengung von Kalziumkristallen wird bei Kaninchen und Meerschweinchen noch als physiologisch angesehen (nahrungsabhängige Aufnahme und renale Elimination). Diese Kristalle entstehen durch eine übermäßige Kalziumzufuhr und/oder eine Störung des Kalziumstoffwechsels (Hyperparathyreoidismus [2], 


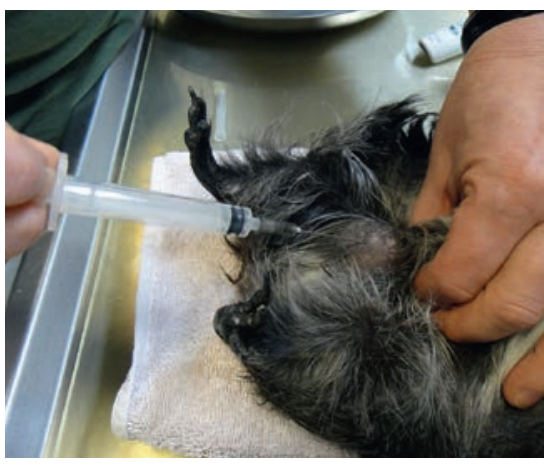

Abb. 3 Zystozentese beim Meerschweinchen (blind).

Osteodystrophia fibrosa [9]). Für die Ausfällung sind unterschiedlichste Faktoren verantwortlich, wie die Anwesenheit von Kristallisationskernen, der pH-Wert, Schleimhautschäden, genetische Prädisposition, Flüssigkeitsaufnahmemenge, Grundkrankheiten etc. Sind zudem Infektionen und/oder entsprechende Milieuveränderungen vorhanden, ist auch die Entstehung von Steinen möglich [12]. Starke Kristallansammlungen und Verfärbungen sollten daher in jedem Fall weiter abgeklärt und therapeutisch (und v.a. diätetisch) angegangen werden.

\section{Urinspezifisches Gewicht (USG)}

Das USG zeigt die Konzentration löslicher Stoffe im Urin an und gibt Hinweise auf die Rückresorptionsfähigkeit des Tubulussystems und somit die Konzentrationsfähigkeit der Nieren. Die Bestimmung erfolgt mittels Refraktometer. Teststreifenmethoden sind aufgrund ungeeigneter Meßbereiche (1000-1025) und schlechter Vergleichbarkeit nicht geeignet [1].

Bei physiologischer Nierenfunktion besteht eine umgekehrte Proportionalität zwischen Konzentration und Urinmenge, d. h. bei geringer Urinmenge ist eine hohe Konzentration zu erwarten und umgekehrt [10]. Bei einem gesunden Kleinsäuger mit intakter Nierenfunktion sollte das USG oberhalb des isostenurischen Bereichs (1008-1016) liegen. Den konzentriertesten Urin der Kleinsäuger haben Hamster und Wüstenrennmäuse mit einem USG von über 1040. Bei Kaninchen und Meerschweinchen wird häufiger ein niedrigeres USG (meist <1025) verzeichnet. Dies ist durch Kalzurie, leichte Infektionen und entsprechende

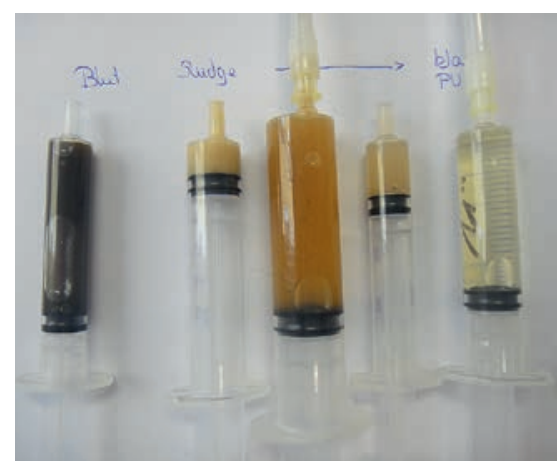

Abb. 4 Urine mit unterschiedlicher Färbung.

Interferenz an den ADH-Rezeptoren der Sammelrohre bedingt. Bei starker Urintrübung sollte der Überstand nach kurzem Stehenlassen verwendet werden.

\section{Urinteststreifen}

Urinteststreifen ( $\bullet$ Tab.2) sind semiquantitive Trockenchemiesysteme zur Bestimmung verschiedener Parameter. Die Durchführung ist schnell und einfach. Die Teststreifenfelder werden mit Urin beträufelt und nach 60 Sekunden (Leukozytenfeld nach 120 Sekunden) abgelesen. Die Auslesung erfolgt visuell und/oder maschinell mit entsprechenden Lesegeräten. Ergebnisse unterschiedlicher Tests und Ausleseverfahren können stark variieren [1].

Urinteststreifen liefern gerade bei kritischen Patienten bereits in der Praxis schnell und einfach erste Aussagen über Stoffwechsellage und Säure-BasenHaushalt des Tieres (pH-Wert, Glukose, Ketonkörper), geben Hinweise auf Infektionen (Leukozyten, Nitrit) und ermöglichen die Unterscheidung von Urinverfärbungen (Blut, Hämoglobin, Myoglobin, Bilirubin oder andere Farbstoffe). Im Idealfall sollten die Urinteststreifenfelder (mit Ausnahme des pH-Wertes) negativ ausfallen.

Der pH-Wert richtet sich nach der Tierart, der Nahrung und dem Gesundheitszustand. Kleinnager haben zumeist einen leicht alkalischen Urin. Diäten mit hohem Gehalt an tierischen Proteinen und katabolische Störungen (z. B. Hunger, Ketose und Fieber) führen zu einer Ansäuerung, pflanzliche Diäten zu einer Alkalisierung [3].

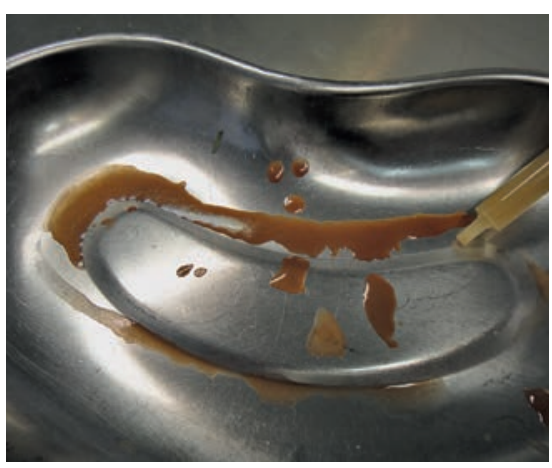

Abb. 5 Nachdunkeln von Urin durch Futterfarbstoffe.

Aufgefangener Urin ist häufig bakteriell kontaminiert, was positive Befunde vortäuschen kann. Besteht laut Urinteststreifen Verdacht auf eine bakterielle Zystitis (Leukozyten, Nitrit), sollte das Ergebnis durch eine Sedimentuntersuchung aus Zystozenteseurin verifiziert und - spätestens bei einem Rezidiv - die Keimart und ihre Empfänglichkeit mittels bakteriologischer Untersuchung und Antibiogramm bestimmt werden.

Der Proteingehalt im Urin korreliert mit dem Blutgehalt und dieser wiederum mit der Art der Uringewinnung. Bei Blasenmanipulation steigt der Blutgehalt (Ausdrücken: 1+ bis 4+ Blut; Zystozentese und Katheterisierung: meist 1+ Blut) und muss entsprechend kritisch bewertet werden.

Glukose kann bei kurzzeitigen Blutglukoseschwankungen (Nahrung, Stress, Östrus) im Urin vorgefunden werden. Der Nachweis von Glukose kann aber auch ein Hinweis auf eine pathologische Hyperglykämie oder Niereninsuffizienz sein und sollte daher kontrolliert und mittels Glukose- und Fruktosaminmessung im Blut verifiziert werden.

Der Nachweis von Ketonkörpern im Urin (nur Azeton und Acetessigsäure) weist auf eine schwerwiegende Stoffwechselentgleisung hin. Die Ursache kann eine Mangelsituation („Hungerketose“) oder wie oft beim Kaninchen eine kurzzeitige Hyperglykämie („diabetische Ketoazidose“) sein. Eine Hypoglykämie konnte bei einer Studie an 907 Kaninchen nur bei $1,4 \%$ festgestellt werden [5]. Die Therapie ist entsprechend abhängig von der Glukosekonzentration in Blut. 


\section{.konkret}

Für die Bestimmung des urinspezifi-

schen Gewichts sind Urinteststreifen nicht geeignet [1].

Da auch Medikamente und Desinfektionsmittel Einfluss auf die Testfelder haben können und so immer sowohl falsch-positive als auch falsch-negative Ergebnisse möglich sind, sollten unplausible Ergebnisse immer durch entsprechende weiterführende Untersuchungen
(Sedimentuntersuchung, Blutuntersuchung, Ultraschall) verifiziert werden [1, 10].

\section{Sedimentuntersuchung}

Die Sedimentuntersuchung ermöglicht eine mikroskopische qualitative und quantitative Bestimmung von:

- Zellen

- Zylindern

- Kristallen

- Mikroorganismen (Bakterien, Hefen etc.)
- anderen Beimengungen (z.B. Farbstoffe, Präzipitate, Zellreste etc.)

Die Uringewinnungsmethode ist hierbei abhängig von der Fragestellung (z.B. Blutung: freiwillig abgesetzter Urin; Verdacht auf bakterielle Zystitis: Zystozenteseurin, - Tab. 1). Zur Sedimentherstellung wird der Urin in einem sterilen Röhrchen (ohne Stabilisator) ca. 5 Minuten bei $400 \mathrm{~g} \mathrm{(z.B.} 1500 \mathrm{U} / \mathrm{min}$. bei $15 \mathrm{~cm}$ Radius) zentrifugiert (höhere Umdrehungen zerstören die Zylinder) und der Überstand weitgehend abgegossen.

Tab. 2 Urinteststreifenparameter.

\begin{tabular}{|c|c|c|}
\hline Parameter & Einflüsse & Aussage \\
\hline Bilirubin & $\begin{array}{l}\text { Falsch positiv: Färbung durch Farbstoffe und } \\
\text { Gallepigmente } \\
\text { Falsch negativ: Teststreifenfehler }\end{array}$ & $\begin{array}{l}\text { Nur wasserlösliches, konjugiertes Bilirubin; Kopplung an Dichloranilin } \\
\text { Hinweis auf Hyperbilirubinämie (prä-, intra- oder posthepatisch), bei } \\
\text { Kleinsäugern selten }\end{array}$ \\
\hline Blut & $\begin{array}{l}\text { Falsch positiv: selbst verursachte Blutung durch } \\
\text { Uringewinnung, Reaktion auf Hämoglobin, } \\
\text { Erythrozyten und Myoglobin, körperliche Aktivität, } \\
\text { Vit. C } \\
\text { Falsch negativ: Teststreifenfehler, starke Trübung }\end{array}$ & $\begin{array}{l}\text { Oxydation von Hydroperoxid durch Hämoglobin/Myoglobin } \\
\text { Abhängig von der Art der Uringewinnung (Anzahl der Ausdrück- } \\
\text { versuche, Zystozentese etc.) }\end{array}$ \\
\hline Glukose & $\begin{array}{l}\text { Falsch positiv: Teststreifenfehler } \\
\text { Falsch negativ: hohe Ketonkörperkonzentration, } \\
\text { Vit. C }\end{array}$ & $\begin{array}{l}\text { Glukoseoxidase-Peroxidase-Methode } \\
\text { Hinweis auf Hyperglykämie (auch kurzzeitig iatrogen oder physiolo- } \\
\text { gisch) oder verminderte Rückresorptionskapazität der Nieren } \\
\text { Positive Befunde sollten durch eine Blutuntersuchung (Glukose, } \\
\text { Fruktosamin) bestätigt werden }\end{array}$ \\
\hline Ketonkörper & $\begin{array}{l}\text { Falsch positiv: Interaktion mit Sulfhydryl, } \\
\text { Phenylketonen, Phthaleine; Spuren ggf. noch } \\
\text { physiologisch } \\
\text { Falsch negativ: Teststreifenfehler, Überwiegen von } \\
\text { Buttersäure, starke Trübung }\end{array}$ & $\begin{array}{l}\text { Probe nach Legal; nur Acetessigsäure und Aceton, keine B-Hydroxy- } \\
\text { buttersäure } \\
\text { Hinweis auf gestörten Energiestoffwechsel (Hyper- oder Hypo- } \\
\text { glykämie) } \\
\text { Beurteilung nur gemeinsam mit pH-Wert und Glukosekonzentration }\end{array}$ \\
\hline Leukozyten & $\begin{array}{l}\text { Falsch positiv: Konservierungsmittel, Antibiotika } \\
\text { (Imipenem, Meropenem, Clavulansäure); } \\
\text { Überdeckung bei starker Färbung } \\
\text { Falsch negativ: Bilirubin, Nitrofurantoin, Glukose, } \\
\text { Protein, Antibiotika (Cephalexin, Gentamycin) }\end{array}$ & $\begin{array}{l}\text { Nachweis der Esteraseaktivität von Granulozyten (intakte und lysierte } \\
\text { Leukozyten) } \\
\text { Positive Befunde sollten durch Sediment bestätigt werden }\end{array}$ \\
\hline Nitrit & $\begin{array}{l}\text { Falsch positiv: Trübung } \\
\text { Falsch negativ: nicht-nitritbildende Bakterien (oft), } \\
\text { zu kurze Verweildauer des Urins in der Blase (ideal } \\
\text { Morgenurin), Antibiotikatherapie innerhalb der } \\
\text { letzten } 3 \text { Tage }\end{array}$ & $\begin{array}{l}\text { Griess'sche Probe - Nitritnachweis (indirekter Nachweis nitrat- } \\
\text { abbauender Bakterien (E. coli etc.) } \\
\text { Positive Befunde hinweisend }\end{array}$ \\
\hline pH-Wert & $\begin{array}{l}\text { Abhängig von Fütterung und Lagerung } \\
\text { sauer = proteinreiche Nahrung } \\
\text { alkalisch = pflanzliche Nahrung und Lagerung } \\
\text { (Temperatursenkung, Urease-bildende Bakterien) }\end{array}$ & $\begin{array}{l}\text { Nachweis von Hydroniumionen } \\
\text { Hinweisend auf Säure-Basen-Haushalt und Stoffwechselsituation }\end{array}$ \\
\hline Protein & $\begin{array}{l}\text { Falsch positiv: alkalischer Urin, Interaktion mit } \\
\text { Desinfektionsmittel, Chlorhexidin } \\
\text { Falsch negativ: sehr dünner und sauer Urin }\end{array}$ & $\begin{array}{l}\text { Proteinreaktion des pH-Indikators (sensitiv auf Albumin) } \\
\text { Abhängig von Gewinnungsart (Blutbeimengung), Grad der Hämaturie } \\
\text { und Urinkonzentrierung (USG) } \\
\text { Hinweis auf renale, urogenitale oder andere Proteinbeimengung } \\
\text { (Blut, Hb, Myoglobin), ggr. Mengen auch bei Fieber, Hypo-/Hyper- } \\
\text { thermie, Stress, Anstrengung }\end{array}$ \\
\hline Urobilinogen & irrelevant beim Tier & $\begin{array}{l}\text { Diazoniumsalzreaktion } \\
\text { Keine klinische Bedeutung beim Tier }\end{array}$ \\
\hline
\end{tabular}


Ein Tropfen des resuspendierten Sediments wird auf einen Objektträger gegeben, mit einem Deckglas abgedeckt und bei geschlossener Irisblende oder heruntergeklapptem Kondensor mikroskopiert $(400 \times)$. Zur besseren Zelldifferenzierung können getrocknete Urinsedimente wie Blutausstriche gefärbt werden (z.B. mit einer Schnellfärbung wie Diff Quick ${ }^{\circledR}$ ). Die Untersuchung sollte zeitnah erfolgen, Standzeit und Lagerung müssen bei der Beurteilung berücksichtigt werden.

Eine Konkrementanalyse kann vom Labor durchgeführt werden, ist bei pflanzenfressenden Kleinsäugern mit alkalischem Urin aber wenig hilfreich. Bei Kaninchen bestehen die Kristalle zu mehr als 90\% aus Kalziumkarbonaten („Calcite“, Korn-, Hantel- oder Biskuitform, > Abb.6), weniger als $3 \%$ bestehen aus Kalziumphosphaten (Schollen- oder große Lattenform, Verwechslung mit Struvitkristallen möglich) und nur selten werden Kristalle aus Ammonium-Magnesium-Phosphaten (Struvit 1,2\%, „Sargdeckelform") oder Kalziumoxalaten $(0,8 \%$, briefumschlagartig [Weddelit] oder seltener zugespitzt, stabförmig [Whewellit]) nachgewiesen [7]. Bei Meerschweinchen überwiegen ebenfalls die Kalziumkarbonate, gerade bei Urethrasteinen scheint der Anteil an Struvit (Beteiligung von Urease-produzierenden Bakterien) aber höher zu sein [4]. Kalziumkarbonate und -oxalate können nicht in der Blase aufgelöst werden, sind nur durch Entfernung (Hydropulsion, Spülung, Zystotomie) behandelbar und dauerhaft nur durch konsequente Umstellung auf kalziumarme Fütterung und Kontrolle zu verhindern.

\section{Erregernachweis}

Die bakteriologische Untersuchung (BU) des Urins dient nicht nur der Verifizierung eines positiven Bakteriennachweises auf Teststreifen oder im Sediment, sondern auch der Keimzahlbestimmung und der Keimdifferenzierung (mit Antibiogramm zum gerichteten Antibiotikaeinsatz). Die Durchführung einer BU ist nur aus steril entnommenem Zystozenteseurin (Versand in sterilen Röhrchen mit Stabilisator) sinnvoll. Bei Verwendung von kontaminier- tem Urin, falscher Lagerung und/oder langem Transport sind falsch-positive Befunde häufig. Falsch-negative Befunde treten während oder innerhalb von einer Woche nach antibiotischer Therapie oder Vorliegen anderer Hemmstoffe auf.

Beim Kleinsäuger gibt es nur wenige andere Erreger, die bei einer Infektion im Urin nachweisbar sind (z. B. Leptospiren, mikroskopisch). Selbst der Nachweis von Encephalitozoon cuniculi-Sporen (Kaninchen etc.) spielt gegenüber der Antikörperbestimmung im Blut (Sensitivität $96 \%$, [6]) nur eine sehr untergeordnete Rolle, da die Sporen nur unregelmäßig beim Platzen von Tubuluszellen frei werden (falsch-negative Befunde: 60\% bei Urin-PCR, 89\% bei Trichromfärbung [8]).

\section{Klinisch-chemische Parameter}

Die Bestimmung des Urin-Protein/Kreatinin-Quotient (UPC) wird bei Hund und Katze mit „sauberem“ Urin (kein Blut, keine Entzündungszellen etc.) zum Nachweis und zur Quantifizierung von Proteinverlust über die Nieren verwendet. Da der Urin von Kleinsäugern aber nur sehr selten klar und ohne Beimengungen ist, ist die Aussagekraft beim Kleinsäuger extrem fraglich. Zudem liegen keine verwendbaren Referenzbereiche vor [1].

Der Urin-Kortisol/Kreatinin-Quotient (UCC) wird vor allem beim Hund zum Ausschluss eines Morbus Cushing verwendet. Aussagekräftige Untersuchungen und Referenzbereiche hierzu liegen bei Kleinsäugern (Meerschweinchen, Hamster, Ratte etc.) noch nicht vor. Der Einsatz beim Frettchen ist nicht zielführend, da der hier vorliegende Hyperadrenokortizismus nicht kortisol- sondern geschlechtshormonbedingt ist.

Die Bestimmung anderer Enzymaktivitäten und Substratkonzentrationen zur Frühdiagnostik von Nierenerkrankungen ist theoretisch auch beim Kleinsäuger möglich, Studien und Referenzbereiche liegen aber bisher nicht vor.

Alle Abbildungen: ㄷ J. Hein

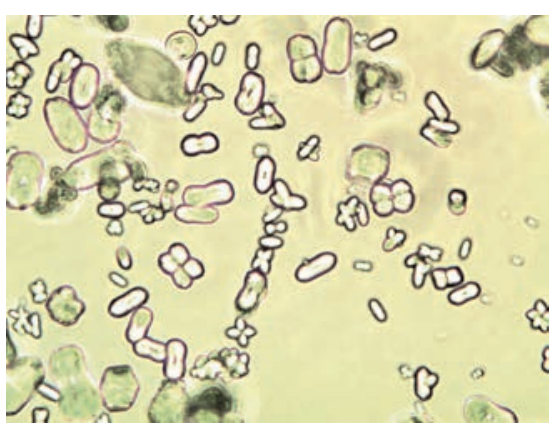

Abb. 6 Polymorphe Kalziumkarbonate im Urinsediment eines Kaninchens (400×).

Online zu finden unter

http://dx.doi.org/10.1055/s-0035-1547402

\section{Literatur}

1 Binder N. Referenzbereiche für Urinparameter bei Kaninchen und Meerschweinchen [Dissertation]. München: LMU München; 2010

2 Buss SL, Bordeau JE. Calcium balance in laboratory rabbits. Miner Electrolyte Metab 1984; 10 (2): 127-132

3 Campell TW. Mammalian hematology: Laboratory animals and miscellaneous species. In: Thrall MA, Weiser G, Allison R, Campbell TW. Veterinary hematology and clinical chemistry. 2nd Philadelphia: Lippincott Williams \& Wilkens; 2012: 211-131

4 Fehr M, Rappold S. Harnsteinbildung bei 20 Meerschweinchen. Tierarztl Prax 1997; 25 : 543-547

5 Harcourt-Brown FM, Harcourt-Brown S. Clinical value of blood glucose measurement in pet rabbits. Vet Rec 2012; 170 (26): 674

6 Hein J, Flock U, Sauter-Louis C, Hartmann K. Encephalitozoon cuniculi in rabbits in Germany - prevalence and sensitivity of antibody testing. Vet Rec 2014; 174 (14): 350

7 Hesse A, Neiger R. Harnsteine bei Kleintieren. Stuttgart: Enke; 2008

8 Jass $A$. Evaluierung von Liquorpunktion und PCR zur klinischen Diagnose der Enzephalitozoonose beim Kaninchen [Dissertation]. München: LMU München; 2004

9 Jordan J, Brunnberg L, Ewringmann A, Müller K. Klinische, radiologische und labordiagnostische Untersuchungen zur Osteodystrophia fibrosa beim Hausmeerschweinchen (Cavia porcellus) der Züchtung „satin“. Kleintierprax 2009; 54: 5-13

10 Moritz A, Schwendenwein I, Kraft W. Harnapparat. In: Kraft W, Dürr UM, Hrsg. Klinische Labordiagnostik in der Tiermedizin. 7. Aufl. Stuttgart: Schattauer; 2014: 420-487 
Tab. 3 Urin: tierartspezifische Besonderheiten (Werte aus $[4,7,11]$ ).

\begin{tabular}{|c|c|c|c|c|c|}
\hline Parameter & Kaninchen & Meerschweinchen & Chinchilla & $\begin{array}{l}\text { Frettchen } \\
\text { (Stinktier) }\end{array}$ & Kleinnager \\
\hline Farbe & $\begin{array}{l}\text { hellgelb bis rotbraun } \\
\text { (Pigmente) }\end{array}$ & $\begin{array}{l}\text { gelb bis rotbraun } \\
\text { (Pigmente) }\end{array}$ & gelb & gelb & hellgelb-gelb \\
\hline Trübung & klar bis leicht trüb & klar bis leicht trüb & klar bis leicht trüb & klar & klar \\
\hline USG & $\begin{array}{l}>1016 \\
\text { (isostenurischer Bereich: } \\
1008-1016 \text { ) }\end{array}$ & $>1016$ & $>1016$ & $>1016$ & $\begin{array}{l}>1016 \text { (Wüsten- } \\
\text { rennmaus u. } \\
\text { Hamster meist } \\
>1040 \text { ) }\end{array}$ \\
\hline $\mathrm{pH}$-Wert & $7,5-9,0(8,25)$ & $8,0-9,0(8,5)$ & $8,0-9,0$ & $6,5-7,5$ & $\begin{array}{l}\text { Maus, Ratte: } 5-7 \\
\text { Hamster, Gerbil: } \\
6-8 \text { (nahrungs- } \\
\text { abhängig) }\end{array}$ \\
\hline Kristalle & $>90 \%$ Kalziumkarbonate & v.a. Kalziumkarbonate & $\begin{array}{l}\text { selten (Ca-Ausschei- } \\
\text { dung v. a. über Darm) }\end{array}$ & v.a. Struvit & selten Struvit \\
\hline
\end{tabular}

11 Quesenberry KE, Orcutt C. Ferrets - Basic approach to veterinary care. In: Quesenberry KE, Carpenter JW, eds. Ferrets, Rabbits, and Rodents: Clinical Medicine and Surgery. 3nd ed. St. Louis: Elsevier; 2012: 13-26
12 Vella D, Donnelly TM. Rabbits - Basic Anatomy, Physiology, and Husbandry. In: Quesenberry KE, Carpenter JW, eds. Ferrets, Rabbits, and Rodents: Clinical Medicine and Surgery. 3nd ed. St. Louis: Elsevier; 2012: 157-173

\section{Dr. Jutta Hein}

Diplomate ECZM (Small Mammal)

Zbz. Heimtiere/Kleinsäuger

Kleintierklinik Augsburg, synlab.vet Augsburg www.heimtieraerztin.de 